\title{
EVOLUTION DES INTENSITES MAXIMALES ANNUELLES DES PLUIES HORAIRES EN CÔTE D'IVOIRE
}

\author{
G. E. SORO'1, B. T. A. GOULA' ${ }^{1}$, F. W. KOUASSI ${ }^{1}$ et B. SROHOUROU ${ }^{2}$ \\ ${ }^{1}$ Laboratoire Géosciences et Environnement, UFR Sciences et Gestion de l'Environnement, Université \\ d'Abobo-Adjamé, 02 BP 801 Abidjan 02, Côte d'Ivoire. E-mail : goulaba2002@yahoo.fr
}

${ }^{2}$ SODEXAM, Direction de la Météorologie Nationale, 15 BP 950, Abidjan 15, Côte d’Ivoire.

\begin{abstract}
RESUME
Les pluies de courtes durées sont indispensables à l'étude d'hydraulique urbaine. En Afrique de l'Ouest, très peu d'études ont été consacrées à l'évolution temporelle des intensités maximales de pluies de courtes durées. Une séries d'intensités maximales annuelles de 8 stations synoptiques ont été analysées sur la période entre1957 et 2001, à l'aide des tests de Mann-Kendall et de régression linéaire. Les résultats montrent une baisse de l'intensité maximale annuelle des pluies de courtes durées. Les pluies de $2 \mathrm{~h}$, $3 \mathrm{~h}$ et $4 \mathrm{~h}$ ont été les plus affectées. Toutefois, la baisse n'a pas été généralisée à l'échelle de la Côte d'Ivoire. Ces résultats ne sont pas suffisants pour établir une relation entre les variations graduelles observées et le phénomène de changement climatique. La complexité du système climatique de l'Afrique de l'Ouest, la variabilité naturelle des données extrêmes et la longueur des séries utilisées suggèrent une prudence en la matière.
\end{abstract}

Mots clés : Pluies extrêmes, détection de tendance, changement climatique, Côte d'Ivoire.

\section{ABSTRACT}

TRENDS IN ANNUAL MAXIMUM INTENSITIES OF HOURLY RAINFALL EVENTS IN CÔTE D'IVOIRE

The rains of short durations are essential in the studies of urban hydraulics. In West Africa, very few studies have been devoted to temporal changes in maximum rain intensities the of short duration. Annual maximum intensity series from 8 stations, over the period spanning from 1957to 2001 were analyzed using the MannKendall and the linear regression tests. Results show a significant decrease in the annual maximum rainfall of short duration. The annual maximum rainfall of $2 h, 3 h$ and $4 h$ durations $n$ were most affected. However, the decrease in annual maximum intensities did not occur everywhere in Côte d'Ivoire. Therefore, changes observed could not be attributed to the climate change phenomenon. The complexity of climate systems in West Africa, natural variability of extremes rainfall, as well as limited length of rainfall series, suggest less enthusiasm.

Key-words : Extreme rainfall, trend detection, climate change, Côte d'Ivoire.

\section{INTRODUCTION}

Le phénomène du changement climatique à l'échelle de la planète est aujourd'hui reconnu. De plus, son incidence sur l'intensité et la fréquence des pluies est bien accepté par la communauté scientifique (De Toffol et al., 2009). Cependant, à ce jour, aucun signal cohérent, à grande échelle, n'a été détecté sur les précipitations extrêmes et, notamment, sur leurs intensités maximales. En effet, de nombreuses études indiquent une augmentation des pluies extrêmes à travers le monde, notamment en Bulgarie (Bocheva et al., 2009), au Brésil (Sugahara et al., 2008), en Inde (Roy et al., 2004), en Russie (Gruza et al., 1999). Par contre, certaines régions du globe présentent des tendances à la baisse au niveau des extrêmes pluviométriques, notamment dans le Kerala en Inde (Pal et Al-Tabbaa, 2009), dans le sud-ouest et l'ouest de l'Australie (Haylock 
et Nicholls, 2000), en Asie du Sud et dans certaines parties du Pacifique Central (Griffiths et al., 2003).

En Afrique de l'Ouest, des études ont montré une diminution des forts évènements pluvieux (Easterling et al., 2000 ; Groisman et al., 2005). Dans cette région tropicale, caractérisée par une alternance de périodes sèches et humides depuis le début du XX $X^{e}$ siècle, très peu d'études ont porté sur l'évolution de l'intensité des pluies de courtes durées. De telles séries pluviométriques sont plutôt rares dans cette partie du globe, malgré les besoins qui s'y présentent depuis une vingtaine d'années. En effet, les ingénieurs du génie civil ou rural ont besoin de connaître les paramètres (IntensitéDurée-Fréquence) de la pluie probable afin de dimensionner les ouvrages qu'ils projettent ou qu'ils entretiennent. Les autorités politiques ou administratives, quant à elles, ont besoin de ces paramètres pour établir des plans de prévention des risques d'inondation.

Face aux perturbations climatiques engendrées par les activités anthropiques, il est primordial d'étudier les éventuels changements dans les séries de pluies de courtes durées afin de protéger efficacement les populations et les infrastructures.

La présente étude constitue la première phase des investigations sur la stationnarité des pluies extrêmes en Côte d'Ivoire. Elle vise à analyser l'évolution de l'intensité maximale annuelle des pluies horaires sur la période 1957 - 2001.

\section{MATERIEL ET METHODES}

\section{ACQUISITION DE DONNEES}

En Côte d'Ivoire, le réseau d'observations pluviométriques est constitué de 14 stations synoptiques réparties sur l'ensemble du pays (Figure 1). Parmi celles-ci seulement 8 stations, équipées de pluviographes, ont été utilisées dans cette étude (Tableau 1). Le choix de ces postes pluviométriques a été fait en fonction du nombre d'années d'observations (supérieur ou égal à 30 ans), du taux de lacunes (inférieur à $5 \%$ ). Le critère lié à la taille de l'échantillon est basé sur les recommandations de l'Organisation Météorologique Mondiale, qui préconise l'utilisation d'une période minimale d'observation de 30 ans pour toute étude sur l'évolution du climat (OMM, 1994). La présence de lacunes dans les séries pluviométriques concernées est susceptible de biaiser leur analyse. Pour réduire de façon significative ce biais, il a été proposé d'utiliser les stations pluviométriques ayant un taux de données inférieur à $5 \%$. Les données issues de pluviographes, qui couvrent la période de 1957 à 2001 ont été analysées et mises à disposition par la société SODEXAM, Côte d'Ivoire.

Tableau 1 : Caractéristiques des stations pluviométriques utilisées en Côte d'Ivoire.

Characteristics of rainfall stations used in Côte d'Ivoire.

\begin{tabular}{lcccccc}
\hline Station & Latitude & Longitude & $\begin{array}{c}\text { Altitude } \\
(\mathrm{m})\end{array}$ & $\begin{array}{c}\text { Période } \\
\text { d'observations }\end{array}$ & $\begin{array}{c}\text { Données } \\
\text { disponibles } \\
(\text { ans })\end{array}$ & $\begin{array}{c}\text { Données } \\
\text { manquantes } \\
(\%)\end{array}$ \\
\hline Abidjan & $5^{\circ} 15 \mathrm{~N}$ & $3^{\circ} 56 \mathrm{~W}$ & 7 & $1958-2001$ & 42 & 4,5 \\
Bouaké & $7^{\circ} 44 \mathrm{~N}$ & $5^{\circ} 40 \mathrm{~W}$ & 376 & $1959-2001$ & 42 & 2,3 \\
Bondoukou & $8^{\circ} 03 \mathrm{~N}$ & $2^{\circ} 47 \mathrm{~W}$ & 371 & $1970-2000$ & 30 & 3,2 \\
Dimbokro & $6^{\circ} 39 \mathrm{~N}$ & $4^{\circ} 42 \mathrm{~W}$ & 92 & $1968-1999$ & 31 & 3,1 \\
Man & $7^{\circ} 24 \mathrm{~N}$ & $7^{\circ} 31 \mathrm{~W}$ & 340 & $1957-2001$ & 43 & 4,4 \\
Korhogo & $9^{\circ} 25 \mathrm{~N}$ & $5^{\circ} 37 \mathrm{~W}$ & 381 & $1971-2001$ & 30 & 3,2 \\
Odienné & $9^{\circ} 30 \mathrm{~N}$ & $7^{\circ} 34 \mathrm{~W}$ & 434 & $1969-2001$ & 33 & 0 \\
Tabou & $4^{\circ} 25 \mathrm{~N}$ & $7^{\circ} 22 \mathrm{~W}$ & 20 & $1964-1998$ & 34 & 2,8 \\
\hline
\end{tabular}




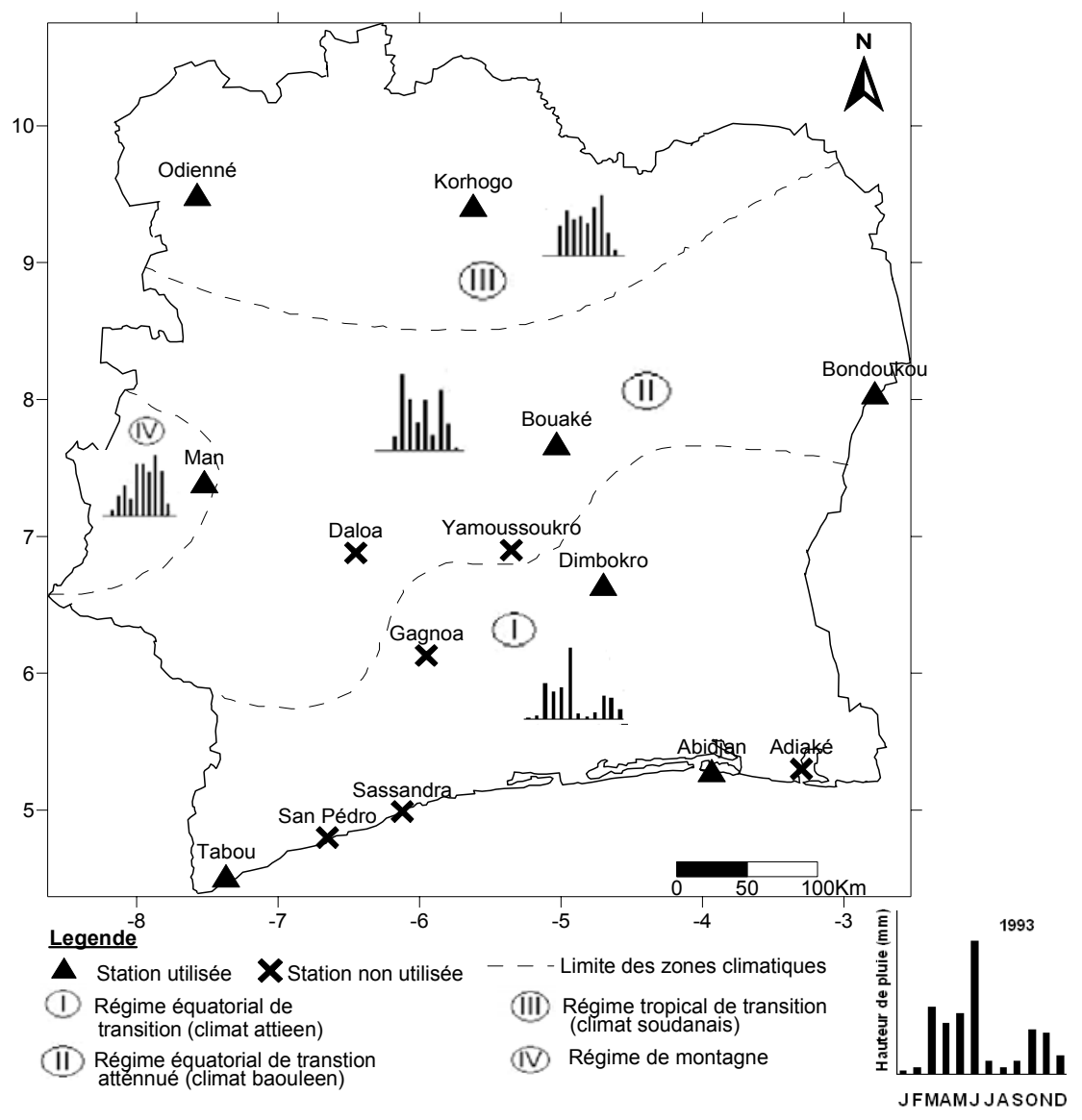

Figure 1 : Localisation des stations synoptiques et des principales zones climatiques de la Côte d'Ivoire.

Localization of the synoptic stations and the principal climatic areas of Côte d'Ivoire.

Deux types de pluviogramme ont été utilisés. L'un gradué en pas de15 min et l'autre en pas de 5 min. Le dépouillement des données a été assuré manuellement selon les pas de temps suivants : $15 \mathrm{~min}, 30 \mathrm{~min}, 45 \mathrm{~min}, 1 \mathrm{~h}, 1 \mathrm{~h}$ $30 \mathrm{~min}, 2 \mathrm{~h}, 3 \mathrm{~h}$ et $4 \mathrm{~h}$.

La variable descriptive (I.maxan) a été utilisée pour caractériser l'intensité des pluies au pas de temps horaire. Cette variable correspond à l'intensité de pluie la plus élevée au cours d'une année donnée.

\section{VERIFICATION DES HYPOTHESES DE BASE}

Pour mener à bien toute étude sur la détection de changements graduels ou brusques dans les séries climatiques, Kundzewicz et Robson (2004) ont proposé la démarche suivante : préparation d'un jeu de données convenable ; analyse exploratoire ; mise en œuvre de tests statistiques appropriés et interprétation des résultats. Ces tests statistiques nécessitent la vérification de quelques hypothèses de base.

\section{Test non paramétrique de Wald- Wolfowitz}

Le test non paramétrique de Wald-Wolfowitz (Wald et Wolfowitz, 1943) a été utilisé pour vérifier l'autocorrélation des séries de variables extrêmes. L'hypothèse de normalité étant rarement vérifiée par les variables climatiques extrêmes, il a été proposé de transformer leur distribution marginale par la technique dite du «Normal scores». Le test non paramétrique «Kendall's turning point» a été utilisé pour vérifier le caractère aléatoire des séries horaires extrêmes. 


\section{Test de Mann-Kendall}

Le test de Mann-Kendall (MK) a permis de détecter d'éventuels changements graduels dans les séries de variables extrêmes. Selon Mann (1945) et Kendall (1975), ce test non paramétrique, basé sur le rang permet de déterminer si la corrélation entre le temps et la variable d'étude est significative ou pas.

Soit $\left(x_{p}, \ldots, x_{n}\right)$ un échantillon de valeurs indépendantes relative à une variable aléatoire $X$ dont on cherche à évaluer la stationnarité.

La statistique de Mann-Kendall est définie comme suit :

$$
S=\sum_{i=1}^{n-1} \sum_{j=i+1}^{n} \operatorname{signe}\left(X_{i}-X_{j}\right)
$$

Où $X_{i}$ et $X_{i}$ sont les valeurs séquentielles des données et $n$ la taille de l'échantillon.

La statistique du test est calculée en dénombrant, pour tous les couples $\left(x_{1}, x_{j}\right)_{i<j}$, le nombre de cas où la seconde valeur est supérieure à la première, et le nombre de cas où la seconde est inférieure, puis en faisant la différence entre ces deux quantités. La présence d'une tendance statistiquement significative est évaluée en utilisant la valeur de $Z$ :

$$
Z=\left\{\begin{array}{l}
\frac{S-1}{\sqrt{\operatorname{var}(S)}}, S>0 \\
0, S=0 \\
\frac{S+1}{\sqrt{\operatorname{var}(S)}}, S<0
\end{array}\right.
$$

En présence de valeurs identiques dans la série, la variance $S$ peut être définie comme suit :

$$
\operatorname{Var}(s)=n(n-1)(2 n+5)-\sum_{i=1}^{n} t_{i} i(i-1) / 18
$$

Où, $t_{i}$ désigne le nombre d'égalités impliquant $k$ valeurs.

Une valeur positive (ou négative) de $Z$ indique une tendance ascendante (ou descendante) et sa significativité est comparée à la valeur critique $\alpha$ ou seuil de significativité du test.

\section{Test de régression linéaire}

Le test de régression linéaire vérifie l'hypothèse de stationnarité contre celle d'une tendance linéaire.

II s'appuie sur un modèle de régression simple entre la série des extrêmes et le temps, défini comme suit :
$X_{t}=\alpha+\beta t+\varepsilon_{t}$

Où $X_{t}$ est la série temporelle étudiée; $t$ est la durée ; est la série des résidus ; $\beta$ est le coefficient de la régression linéaire.

Par la méthode des moindres carrées, les paramètres sont estimés de la manière suivante:

Où $\bar{X}$ et $\bar{t}$ sont respectivement la moyenne de la série étudiée et de la période d'observation; $\hat{\alpha}$ et $\hat{\beta}$ sont respectivement les estimateurs de et .

La variance de l'estimateur de tendance peut être estimée par :

Où $n$ est la taille de l'échantillon (série temporelle).

Ce test consiste alors à vérifier que l'estimateur du coefficient directeur $\beta$ est proche de zéro. Pour cela, on compare la statistique suivante aux quantiles d'une loi de Student à $(n-2)$ degrés de liberté :

II s'agit d'un test paramétrique, qui suppose la normalité des données, et est spécialement conçu pour détecter une tendance linéaire.

\section{Procédure Bootstrap}

La procédure de re-échantillonnage Bootstrap a permis d'estimer le niveau de significativité des tests de Mann-Kendall et de régression linéaire. Les détails concernant la procédure de reéchantillonnage Bootstrap sont donnés par Lettenmaier et al. (1994).

\section{Indice de tendance}

Les indices de tendance sont utilisés pour présenter les résultats relatifs aux tests de Mann-Kendall et de régression linéaire. L'indice de tendance IT $(\%)$ est lié au niveau de significativité $\alpha(\%)$ des tests statistiques. Les 
indices de tendance sont définis comme suit (Kundzewicz et al., 2005) :

$$
\begin{aligned}
& \text { pour les tendances positives } \\
& \text { pour les tendances négatives }
\end{aligned}
$$

La valeur de l'indice de tendance varie entre $-100 \%$ et $+100 \%$. Les valeurs négatives correspondent aux tendances à la baisse et les valeurs positives aux tendances à la hausse. Une tendance significative à la baisse au risque de $10 \%$ aura un indice de tendance de $-90 \%$.

La détection des tendances et l'estimation du niveau de significativité des tests statistiques ont été réalisées à l'aide du logiciel HYDROSPECT.

\section{RESULTATS}

\section{DEPENDANCE SERIELLE}

Dans l'ensemble, les séries pluviométriques horaires n'ont pas présenté d'autocorrélation significative, à l'exception de celles des stations d'Abidjan, de Dimbokro, Man et Tabou (Tableau 2). Au niveau de la station d'Abidjan, le phénomène de dépendance a été fortement $I T= \begin{cases}100-\alpha & \text { ressenti au niveau des pas de temps compris }\end{cases}$ centre $1 \mathrm{~h}$ et $2 \mathrm{~h}$. Au niveau de la station de Dimbokro, les séries d'intensités, au-delà de 45 min, ont été affectées par une autocorrélation positive. Certaines séries ont présenté une structure d'autocorrélation négative, mais celleci n'a pas été significative au risque de $5 \%$. Toutes les structures d'autocorrélation négative (5 pour toutes les 8 stations) ont présenté de très faibles coefficients.

\section{EVOLUTION DES SERIES HORAIRES}

Les indices de tendance (IT) négatifs indiquent une tendance à la baisse des pluies horaires extrêmes et les indices de tendance positifs présentent une tendance à la hausse (Tableau 3). Dans l'ensemble, $47,7 \%$ des séries ont été affectées par une tendance significative à la baisse. Les stations les plus affectées par cette modification graduelle ont été celles de Tabou, de Dimbokro et d'Abidjan. Dans ces stations, les durées d'agrégation au-delà de 45 min ont été les plus sensibles à cette modification graduelle. Dans les stations d'Abidjan et de Dimbokro, la majorité des tendances significatives ont été détectées dans des séries présentant une structure d'autocorrélation positive.

Au niveau des stations d'Abidjan, Bondoukou, Dimbokro et d'Odienné, les tendances significatives détectées par le test de MannKendall ont été identiques à celles détectées par le test de régression linéaire. Cela s'est traduit par une très forte corrélation entre les indices de tendance des deux tests statistiques (Tableau 4).

Cette forte corrélation des indices de tendance confirme l'existence de changements graduels au niveau des pluies horaires extrêmes dans ces stations. A la station de Man, les indices de tendance issus du test de Mann-Kendall diffèrent de ceux relatifs à la régression linéaire. Cette différence est caractérisée par un coefficient de corrélation très faible 0,02 . Pour les pluies de durée $1 \mathrm{~h}$, la corrélation entre le temps et l'intensité maximale annuelle a été très faible (Figure 2). En effet, pour cette durée d'agrégation, le coefficient de corrélation n'a pas excèdé 0,3. Certaines séries (Abidjan, Dimbokro, Tabou et Korhogo) indiquent des tendances à la baisse. Par contre, d'autres n'ont présenté aucune tendance (Man, Odienné, Bondoukou). La série de Bouaké a présenté une très légère tendance à la hausse.

\section{PERFORMANCES DES TESTS DE MANN- KENDALL ET DE REGRESSION LINEAIRE}

La performance, en terme de nombre de tendances détectés des tests de Mann-Kendall et de régression linéaire, a été évaluée. On observe une légère supériorité du test de MannKendall par rapport au test de régression linéaire (Figure 3). En effet, pour des niveaux de significativité de $5 \%$ et $10 \%$, le test MannKendall semble être plus puissant à détecter des tendances que le test de régression linéaire. 
Tableau 2 : Coefficient d'autocorrélation d'ordre 1 des intensités maximales annuelles des pluies de courtes durées observées en Côte d'Ivoire.

Coefficient of autocorrelation of order 1 of the annual maximum rainfall intensities of short duration as observed in Côte d'lvoire.

\begin{tabular}{lcccccccc}
\hline \multirow{2}{*}{ Station } & \multicolumn{7}{c}{ Coefficient d'autocorrélation d'ordre 1 } \\
\cline { 2 - 8 } & $15 \mathrm{~min}$ & $30 \mathrm{~min}$ & $45 \mathrm{~min}$ & $1 \mathrm{~h}$ & $1 \mathrm{~h} 30 \mathrm{~min}$ & $2 \mathrm{~h}$ & $3 \mathrm{~h}$ & $4 \mathrm{~h}$ \\
\hline Abidjan & $-0,04$ & 0,05 & 0,20 & 0,40 & 0,40 & 0,30 & $-0,07$ & 0,04 \\
Bouaké & 0,08 & 0,15 & 0,16 & 0,12 & 0,05 & 0,00 & 0,00 & 0,03 \\
Bondoukou & $-0,07$ & 0,11 & 0,27 & 0,12 & 0,02 & 0,10 & 0,21 & 0,20 \\
Dimbokro & 0,15 & 0,19 & 0,26 & 0,37 & 0,35 & 0,31 & 0,32 & 0,31 \\
Korhogo & $-0,08$ & 0,12 & 0,04 & 0,05 & 0,06 & 0,19 & 0,22 & 0,22 \\
Man & 0,09 & 0,28 & 0,19 & 0,31 & 0,26 & 0,27 & 0,20 & 0,15 \\
Odienné & 0,24 & 0,21 & 0,07 & 0,12 & 0,10 & 0,20 & 0,07 & 0,01 \\
Tabou & 0,33 & 0,22 & 0,05 & $-0,01$ & 0,15 & 0,21 & 0,25 & 0,24 \\
\hline
\end{tabular}

Les chiffres en italique indiquent une autocorrélation significative au risque $5 \%$ )

The figures in italic indicate significant autocorrelation at $5 \%$ level the risk

Tableau 3 : Indices de tendance des séries pluviométriques de courtes durées observées en Côte d'Ivoire. Trend index in rainfall series of short duration observed in Côte d'lvoire.

\begin{tabular}{lcccccccc}
\hline \multirow{2}{*}{ Test } & \multicolumn{7}{c}{ Indices de tendance $(\%)$} \\
\cline { 2 - 8 } & $15 \mathrm{~min}$ & $30 \mathrm{~min}$ & $45 \mathrm{~min}$ & $1 \mathrm{~h}$ & $1 \mathrm{~h} 30 \mathrm{~min}$ & $2 \mathrm{~h}$ & $3 \mathrm{~h}$ & $4 \mathrm{~h}$ \\
\hline MK & $-75,8$ & $-98,2$ & $-95,7$ & $-98,9$ & $-98,7$ & $-97,8$ & $-98,6$ & $-97,5$ \\
RL & $-2,6$ & $-76,7$ & $-83,8$ & $-98,4$ & $-98,5$ & $-96,1$ & $-98,3$ & $-97,5$ \\
\hline MK & $-39,3$ & 67,5 & 45,9 & 27,5 & 3,2 & 52 & 62,3 & 49,9 \\
RL & $-68,7$ & 34,4 & 29,4 & 18,6 & 0,4 & 33,9 & 29,1 & 11,4 \\
\hline MK & 98,5 & 78,9 & 70,9 & 12,2 & 52,7 & $-91,9$ & $-96,9$ & $-97,5$ \\
RL & 87,7 & 80,5 & 82,5 & 7,6 & 60,3 & $-89,7$ & $-94,5$ & $-95,3$ \\
\hline MK & $-82,7$ & $-98,1$ & $-98,5$ & $-99,3$ & $-98,8$ & $-99,1$ & $-98,8$ & $-99,1$ \\
RL & $-60,8$ & $-98,9$ & $-99,7$ & $-99,5$ & $-99,2$ & $-99,1$ & $-98,3$ & $-98,6$ \\
\hline MK & $-93,7$ & $-86,3$ & $-91,4$ & $-60,9$ & 1,74 & 25,7 & 24,6 & 29,4 \\
RL & $-73,2$ & $-61,7$ & $-61,2$ & $-35,8$ & 10,3 & 27,1 & 13,1 & 23,6 \\
\hline MK & $-90,3$ & $-90,1$ & $-66,8$ & $-77,6$ & $-66,8$ & $-95,5$ & $-99,5$ & $-99,2$ \\
RL & $-96,5$ & $-94,3$ & $-75,5$ & $-72,6$ & $-84,5$ & $-96,1$ & $-97,6$ & $-98,9$ \\
\hline MK & $-89,8$ & $-44,3$ & $-38,2$ & $-51,1$ & $-39,7$ & $-79,8$ & $-91,7$ & $-93,5$ \\
RL & 76,2 & $-38,2$ & $-21,2$ & $-20,1$ & $-52,2$ & $-67,5$ & $-91,7$ & $-91,2$ \\
\hline MK & $-82,7$ & $-98,1$ & $-98,5$ & $-99,3$ & $-98,8$ & $-99,1$ & $-98,8$ & $-99,1$ \\
RL & $-60,8$ & $-98,9$ & $-99,7$ & $-99,5$ & $-99,2$ & $-99,1$ & $-98,3$ & $-98,6$ \\
\hline
\end{tabular}

Les chiffres en italique indiquent les tendances significatives au risque de $10 \%$; MK : Mann-Kendall ; RL : régression linéaire.

The figures in italic indicate significant trends at level of risk $10 \%$; MK : Mann-Kendall ; RL : linear regression. 

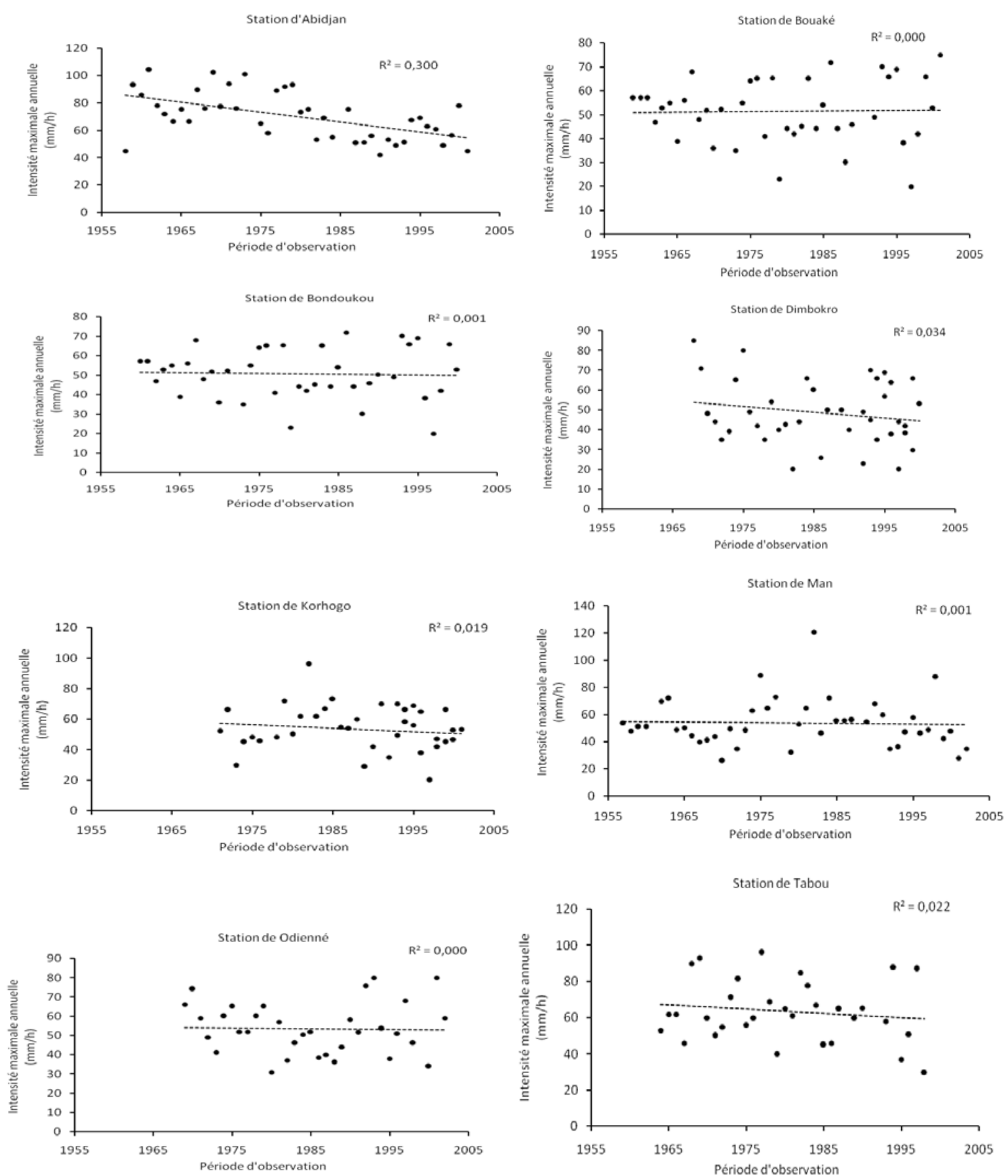

Figure 2 : Evolution des intensités maximales annuelles de pluies de durée d'agrégation de 1 heure pour différentes stations synoptiques en Côte d'Ivoire.

Evolution of annual rainfull maximum intensities of 1 hour duration on aggregate regarding different synoptic stations in Côte d'Ivoire. 
Tableau 4 : Coefficient de corrélation des Indices de tendance relatif aux tests de Mann-Kendall et de régression linéaire pour différentes stations pluviométriques en Côte d'Ivoire.

Correlation coefficient of trend index related to Mann-Kendall and linear regression tests for different rainfall stations in Côte d'Ivoire.

\begin{tabular}{lc}
\hline Station & $\begin{array}{c}\text { Coefficient de corrélation } \\
\text { des indices de tendance }\end{array}$ \\
\hline Abidjan & 0,95 \\
Bouaké & $*$ \\
Bondoukou & 0,99 \\
Dimbokro & 0,99 \\
Korhogo & $*$ \\
Man & 0,02 \\
Odienné & 0,99 \\
Tabou & 0,74 \\
${ }^{*}:$ absence de tendance significative dans toutes les séries de la \\
station. \\
*: no significant trend regarding all series.
\end{tabular}

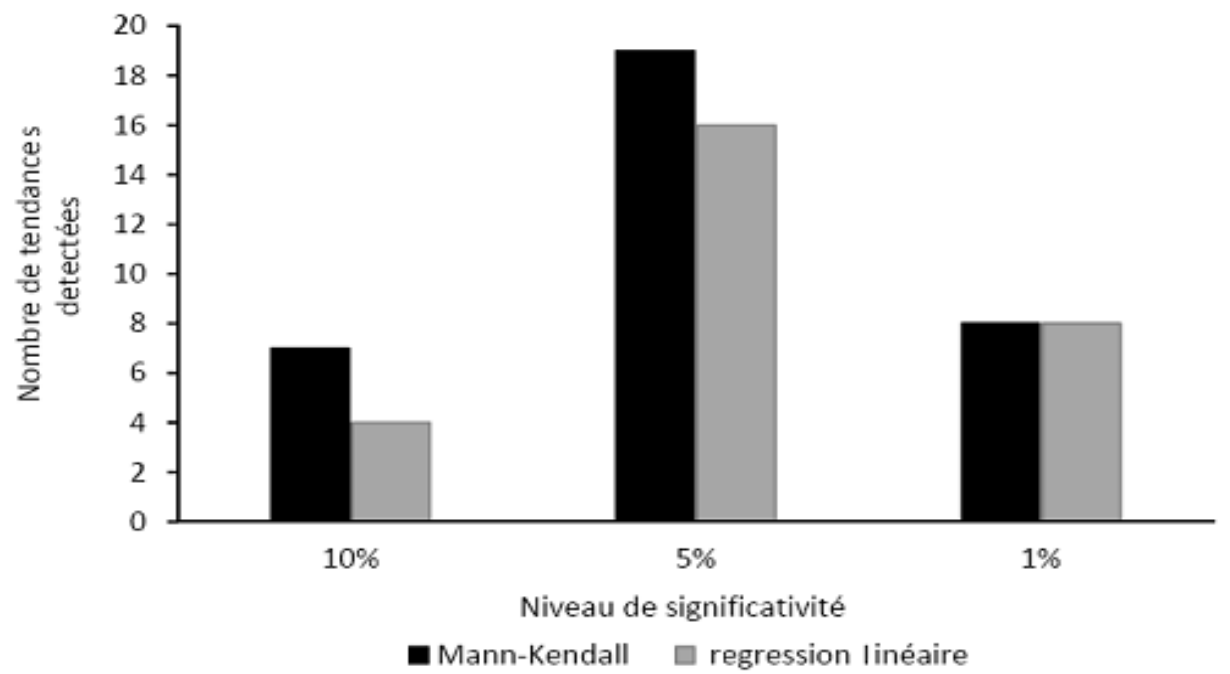

Figure 3 : Tendances détectées selon trois seuils de significativité et les tests utilisés.

Detected trends following three levels of significance as well as used.

\section{COHERENCE SPATIALE DES TENDANCES}

L'approche descriptive est utilisée pour dégager d'éventuelles cohérences spatiales des changements locaux détectés. Les tendances présentées dans cette partie sont celles détectées par le test de Mann-Kendall. Les tendances détectées au niveau des différents pas de temps indiquent une baisse de l'intensité maximale des pluies de courtes durées (Figure 4). En outre, l'on constate que le nombre de tendances détectées augmente avec la durée de la pluie. En effet, la baisse de l'intensité est fortement ressentie au niveau des pluies de durées comprises entre $2 \mathrm{~h}$ et $4 \mathrm{~h}$. Les pluies de durée inférieure à $2 \mathrm{~h}$ présentent très peu de changements graduels à la baisse. Pour les pluies de durée $15 \mathrm{~min}$, l'on a observé une tendance à la hausse au niveau de la station de Bondoukou et une tendance à la baisse au niveau de la station de Korhogo. Les tendances détectées dans les pluies de durées inférieures à $2 \mathrm{~h}$ ne présentent pas de cohérence spatiale. Au niveau des pluies de durées supérieures à $2 \mathrm{~h}$, certaines tendances à la baisse semblent se regrouper au Sud, au Sud-Est, au Nord-Est, à l'Ouest et au Nord-Ouest. 

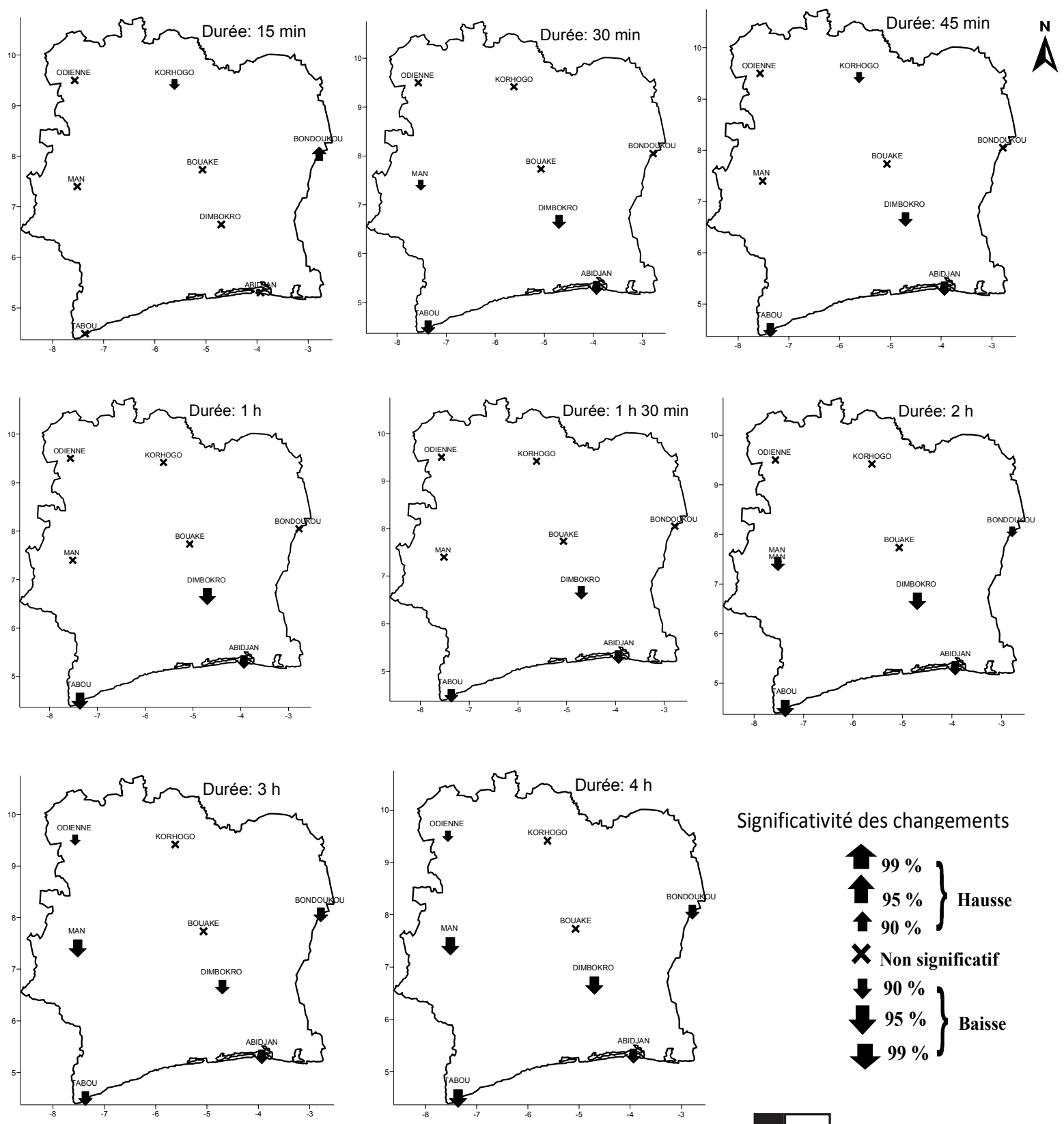

Significativité des changements
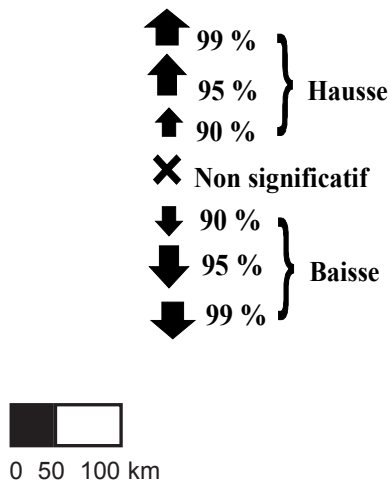

Figure 4 : Tendances au niveau des séries pluviométriques annuelles d'intensités maximales. Trends in annual maximum rainfall intensities. 


\section{DISCUSSION}

\section{PHENOMENE DE DEPENDANCE SERIELLE}

Les résultats des tests statistiques montrent que globalement $50 \%$ des séries d'intensités maximales annuelles de pluie ont été affectées par un changement graduel à la baisse. Dans les séries analysées, très peu ont été affectées par un changement graduel à la hausse. La majorité des changements à la baisse ont été détectées dans des séries présentant une structure d'autocorrélation positive. Dans les séries horaires extrêmes d'Abidjan et de Dimbokro, cette autocorrélation positive a été significative au risque de $5 \%$. Par contre, dans d'autres séries, ce phénomène de dépendance sérielle a été présent, mais n'a pas été significatif au seuil de $5 \%$. Qu'il soit significatif ou pas, la présence d'une structure d'autocorrélation positive, dans les séries de données pluviométriques a semblé perturber considérablement les tests statistiques par l'introduction de tendances apparentes. Cellesci sont souvent dues à la décroissance lente de la fonction d'autocorrélation (Renard, 2006). Dans le cas des séries fortement autocorrélées, les tendances apparentes proviennent rarement d'une évolution déterministe à long terme. Certains auteurs (Yue et Wang, 2004 ; Hamed, 2009) ont indiqué que la présence d'autocorrélations a provoqué un fort taux de rejet de l'hypothèse de stationnarité (absence de tendance). Yue et al. (2002) ont souligné que la présence d'une autocorrélation négative a induit un effet inverse, autrement dit, l'on ne rejette que très peu l'hypothèse de stationnarité des séries horaires extrêmes. Pour remédier au problème lié à l'autocorrélation, Von Storch et Navarra (1999) ont proposé de «blanchir» les observations avant l'application des tests. Yue et al. (2003) ont toutefois critiqué cette procédure en démontrant qu'elle n'était pas une opération neutre lorsque l'autocorrélation et la tendance déterministe sont présentes dans la série à traiter. Jaruskova (1997) suggère une plus grande prudence au niveau de la fonction d'autocorrélation. II a affirmé qu'il est quasiment impossible de préciser qui de l'autocorrélation ou d'une tendance déterministe est responsable d'une apparente non-stationnarité. Les tendances détectées au niveau des pluies de durées inférieures à $2 \mathrm{~h}$ ne présentent pas de cohérence spatiale. Au niveau des pluies de durées supérieures à $2 \mathrm{~h}$, certaines tendances semblent se regrouper au Sud, au Sud-Est, au Nord-Est, à l'Ouest et au Nord-Ouest.

\section{PERFORMANCE DES TESTS STATISTIQUES UTILISES}

Les baisses au niveau de l'intensité maximale des pluies de courtes durées même si elles n'ont pas affecté toutes les stations et tous les pas de temps, ont confirmé néanmoins la tendance générale à la baisse de la pluviométrie en Afrique de l'Ouest (Paturel et al., 1998 ; Servat et al., 1999), notamment en Côte d'Ivoire (Goula et al., 2006).

Au niveau de la performance des tests statistiques utilisés, on a observé une légère supériorité du test de Mann-Kendall, par rapport au test de régression linéaire. En effet, pour des niveaux de significativité de $5 \%$ et $10 \%$, le test Mann-Kendall a semblé plus puissant dans la détection des tendances que le test de régression linéaire. Ces résultats diffèrent de ceux obtenus par Zhang et al. (2004). Ces auteurs affirment que, dans le cas des valeurs extrêmes, le test de régression linéaire est significativement plus puissant que le test de Mann-Kendall.

Hirsch et al. (1991) et Yue et Pilon (2004), ont indiqué que le test de régression linéaire s'avère légèrement supérieure au test de Mann-Kendall si les données sont gaussiennes. Ce qui est contraire à nos résultats. En effet, la majorité des séries d'intensités maximales ont été non gaussiennes. Kundzewicz et Robson (2000) a souligné que la difficulté des tests statistiques à détecter des tendances peut être aussi liée à la forte variabilité naturelle et à la taille des séries pluviométriques extrêmes.

\section{CONCLUSION}

Cette étude consacrée à l'évolution de l'intensité maximale annuelle des pluies de courtes durées s'est déroulée dans 8 stations synoptiques où des données ont été enregistrées pendant au moins 30 ans. L'analyse des séries horaires sur la période 1957 à 2001, à l'aide de tests statistiques a montré une baisse de l'intensité maximale annuelle des pluies de courtes durées. Toutefois, celle-ci n'est pas généralisée à l'échelle de la Côte d'Ivoire. Certaines stations notamment celles situées au Centre et au Nord 
n'ont pas présenté de tendances significatives. Ces résultats ne permettent pas d'attribuer ces changements graduels détectés au changement climatique. II est en effet difficile de discerner cette influence parmi toutes les autres sources de changements possibles. La variabilité naturelle des données extrêmes complique également l'interprétation des variations observées, et les résultats présentés dans cette étude ne suffisent pas pour déterminer leur cause : impact du changement climatique ou variabilité climatique naturelle. Par ailleurs, la longueur des séries d'intensités maximales annuelles aujourd'hui disponibles (inférieure à 50 ans) est insuffisante pour répondre à cette interrogation.

La seconde phase de ce travail qui concerne la stationnarité des pluies journalières extrêmes pourraient nous apporter quelques éléments de réponse à cette interrogation.

\section{REMERCIEMENTS}

Les auteurs remercient la Direction de la Météorologie Nationale de la Côte d'Ivoire et Dr. Maciej Radziejewski pour la mise à disposition des données pluviométriques et du logiciel HYDROSPECT, respectivement.

\section{REFERENCES}

Bocheva L., Simeonova M. P. and I. Gospodinova. 2009. Variability and trends of extreme precipitation events over Bulgaria (1961 2005). Atmos. Res. 93 (1 - 3) : 490 - 497.

De Toffol S., Laghari A. N. and W. Rauch. 2009. Are extreme rainfall intensities more frequent? Analysis of trends in rainfall patterns relevant to urban drainage systems. Water Sci. Technol. 59 (9) : 1769 - 1776.

Easterling D. R., Meehl G. A., Parmesan C., Changnon S. A., Karl T. R. and L.O. Mear. 2000. Climate extremes: observations, modeling, and impacts. Sciences 289 : 2068 - 2074.

Goula B. T. A., Savané I., Konan B., Fadika V. et G. B. Kouadio. 2006. Impact de la variabilité climatique sur les ressources hydriques des basins versants du N'Zo et du N'Zi en Côte d'Ivoire (Afrique tropicale humide). Vertigo 7 (1): 1 - 12.

Griffiths G. M., Salinger M. J. and I. Leleu. 2003. Trends in extreme daily rainfall across the
South Pacific and relationship to the South Pacific convergence zone. Int. J. Climatol. $23: 847-869$.

Groisman P. Y., Knight R. W., Easterling D. R. and T. R. Karl. 2005. Trends in intense precipitation in the climate record. J. Climate. 18 (8) : 1326 - 1349.

Gruza G., Rankova E., Razuvaev V. and O. Buygina. 1999. Indicators of climate chance for the Russian Federation. Climatic change 42 : $219-242$.

Hamed K. H. 2009. Effect of persistence on the significance of Kendall's tau as a measure of correlation between natural time series. Eur. Phys. J. Topics. $174: 65$ - 79.

Haylock M. and N. Nicholls. 2000. Trends in extreme rainfall indices for an updated high quality data set for Australia, 1910 - 1998. Int. J. Climatol. $20: 1533$ - 1541.

Hirsch R. M., Alexander M. B. and R. A. Smith. 1991. Selection of methods for the detection and estimation of trends in water quality. Water Resour. Res. 27 (5) : 803 - 813.

Jaruskova D. 1997. Some problems with application of change-point detection methods to environnement data. Environnemetrics $8: 469-483$.

Kendall M. G. 1975. Rank correlation methods. Griffin, London, 202 p.

Kundzewicz Z. W. and A. Robson. 2000. Detecting trend and Other Changes in Hydrological Data; World Climate Programme-water, World Climate Programme Data and Monitoring, WCDMP-45, World Meteorological Organisation, Geneva, Switzerland, $168 \mathrm{p}$.

Kundzewicz Z. W. and A. Robson. 2004. Change detection in river flow records-review of methodology. Hydrol. Sci. J. 49 (1) : 3 - 6.

Kundzewicz Z. W., Graczyk D., Maurer T., Pinskwar I., Radziewjiewiski M., Svensson C. and M. Szwed. 2005. Trend detection in river flow series : 1. Annual maximum flow. Hydrol. Sci. J. 50 (5) : 797 - 810.

Lettenmaier D. P., Wood, E. F. and J. R. Wallis. 1994. Hydro-climatological trends in the continental United-States, 1948 - 88. J. Climate. $7: 586$ - 607.

Mann H. B. 1945. Non parametric tests against trend. Econometrica $13: 245$ - 259.

OMM. 1994. Guide des pratiques hydrologiques: acquisition et traitement des données, analyses, prévision et autres applications. Organisation Météorologique MondialeNN168, Genève, 829 p. 
Pal I. and A. Al-Tabbaa. 2009. Trends in seasonal precipitation extremes - an indicator of "climate change» in Kerala, India. J. Hydrol. $367: 62$ - 69 .

Paturel J. E., Servat E. et M. O. Delattre. 1998. Analyse de séries pluviométriques de longue durée en Afrique de l'Ouest et centrale non sahélienne dans un contexte de variabilité climatique. Hydrol. Sci. J. 43 (3) : 937 - 945.

Renard B. 2006. Détection et prise en compte d'éventuels impacts du changement climatique sur les extrêmes hydrologiques en France. Thèse de Doctorat ès Science. Institut National Polytechnique de Grenoble (France), $269 \mathrm{p}$.

Roy S. S. and Jr. R. C. Balling. 2004. Trends in extreme daily rainfall indices in India. Int. J. Climatol. 24 : 457 - 466.

Servat E., Paturel J. E., Lubes-Niel H., Kouamé B., Masson J. M., Travaglio M. et B. Marieu. 1999. De différents aspects de la variabilité de la pluviométrie en Afrique de l'ouest et centrale non sahélienne. Rev. Sci. Eau. $12(2)$ : 363 - 387.

Sugahara S., Porfírio da Rocha R. and R. Silveira. 2008. Non-stationary frequency analysis of extreme daily rainfall in Sao Paulo, Brazil. Int. J. Climatol. 29 (9) : 1339 - 1349.
Von Storch H. and A. Navarra. 1999. Analysis of climate variability. Second edition. SpringerVerlag, $364 \mathrm{p}$.

Wald A. and J. Wolfowitz. 1943. An exact test for randomness in the non parametric case based on the serial correlation. Ann. Math. Stat. $14: 378-388$

Yue S., Pilon P. and B. Phinney. 2003. Canadian streamflow trend detection: impacts of serial and cross-correlation. Hydrol. Sci. J. 48 (1) : 51 - 63.

Yue S. and P. Pilon. 2004. A comparison of the test $t$ test, Mann-Kendall and bootsrap test for trend detection. Hydrol. Sci. J. 49 (1) : $21-37$.

Yue S., Pilon P., Phinney B. and G. Cavadias. 2002. The influence of autocorrelation on the ability to detect trend in hydrological series. Hydrological Processes 16 : 1807 - 1829.

Yue S. and C. Y. Wang. 2004. The Mann-Kendall test modified by effective sample size to detect trend in serially correlated hydrological series. Water Ressources Management $18: 201-218$.

Zhang X. B., Zwiers W. F. and G. L. Li. 2004. Monte Carlo experiments on the detection of trends in extremes valeues. J. Climate. 17 : 1945 - 1952. 